\title{
Journalismus ist philosophiebedürftig
}

\author{
Die Medien zwischen Wissenschaft, Moral und Technik
}

\author{
von Hermann Boventer
}

Welche Wirklichkeit bringt eigentlich der Journalismus hervor? Das ist eine philosophische Frage, und sie wollen wir im folgenden zu den Phänomenen des Journalismus in Beziehung setzen. Welche Wirklichkeit bringt zum Beispiel das Fernsehen hervor? Haben wir es mit einer „erfundenen Wirklichkeit“ zu tun, wie Paul Watzlawik behauptet, mit einer Wirklichkeit, die uns nach dem Wissen dessen fragen läßt, „was wir zu wissen glauben "? ${ }^{1}$ Und hätten wir dann mit einem solchen Hinweis ein durchgängiges Konstruktionsprinzip für allen Journalismus in der "Verarbeitung“ der Wirklichkeit benannt?

In John Lockes Erkenntnistheorie gibt es den berïhmten Satz, nichts sei in der Seele, was nicht zuvor in den Sinnen gewesen ist. Wenn die These von der Wirklichkeitskonstruktion durch den Journalismus sich als zutreffend erweisen sollte, dann kann uns der weitere Fortschritt der elektronischen Kommunikation schon bald eine Zukunft bringen, in der John Lockes Satz lauten würde: Es ist nichts in der Seele, was nicht zuvor in den Medien gewesen ist. Zu einer Zeit, als es noch kein Fernsehen gab, hat Karl Kraus diese Entwicklung schon auf die Formel gebracht: „Im Anfang war die Presse, und dann erschien die Welt." Die Frage, wie wirklich die journalistische Wirklichkeit denn tatsächlich ist, dient uns hier als Ausgangspunkt für die weitergehende Fragestellung nach den philosophischen Strukturen des Journalismus, der wir vor allem in ethischer Absicht nachgehen wollen.

Auf einer Karikatur ist ein Vater im Lehnstuhl zu sehen. Der Vater ist in ein Buch vertieft. Da unterbricht ihn sein neugieriger Sohn mit der Frage: „Daddy, wenn ein Baum im Wald umstürzt, aber die Medien sind nicht dabei, um darüber zu berichten, ist dann der Baum wirklich umgefallen?" ${ }^{2}$ Die Medien sind eine Welt. Worüber sie nicht berichten, das „existiert" in dieser Welt der Medien auch nicht. Nach Möglichkeit will man sogar noch vor den Ereignissen liegen. ${ }^{3}$ Die Frage stellt sich, was überhaupt der "wahre" Charakter der journalistischen Realität ist, ob wir es nicht stets mit einer Hypothese zu tun haben, die sich letzten Endes weder falsifizieren noch verifizieren läßt. ${ }^{4}$ Wären dann Journalisten als Personen zu kennzeichnen, die hauptberuflich mit einer spezifischen Unterstellung von Realität handeln?

Das Wissen von der journalistischen Wirklichkeitskonstruktion erfährt seit einigen Jahren eine erhöhte Aufmerksamkeit durch die Wissenschaft. Soweit diese Wissenschaft auf ihr empirisch-analytisches Instrumentarium zurückgreift, sind dem journalistischen Wirklichkeitsbegriff und der Reflexion über ihn enge Grenzen gesetzt. Die Voraussetzung einer Medienrealität wird nicht zum „Problem“, sondern die Wirklichkeit erscheint als ein positivistisch und selbstverständlich Vorgegebenes. Sie ist das Material, gewissermaßen der Werkstoff des Journalismus, woraus er seine Produkte herstellt. Erst neuerdings sind Vorstellungen von der „Konstruktion einer gesellschaftlichen Wirklichkeit" und von einer durch die Medienrealität bedingten Sozialisation des „Wissens, welches das Verhalten in der Alltagswelt reguliert " ${ }^{\text {, }}$, in die kommuni-

D1. phil. Hermann Boventer, Bensberg, ist freier Publizist, und Lehrbeauftragter für politische Pädagogik an der Universität Bonn. 
kationswissenschaftlichen Oberlegungen eingebracht worden, und die komplexe Reziprozität, die im Wirklichkeits- und damit auch im Wirkungsbegriff des Journalismus enthalten ist, findet in erheblich stärkerem Maße eine Berücksichtigung. Die Auffassung, wir hätten es bei der Berichterstattung der Massenmedien mit einer quasi naturwüchsig vorgegebenen Realität zu tun, ist bereits von Walter Lippmann in den frühen zwanziger Jahren mit seinem Hinweis auf die Zwischenwelt der Symbole und Stereotypen problematisiert worden. "Pictures in our heads" sind es, die sich hier in rationalistischer Selbsttäuschung als öffentliche Meinung zu objektivieren suchen, und Lippmann zeigt auch schon sehr eindrucksvoll die moralische Natur des Journalismus. ${ }^{6}$ Lippmanns Fragestellung ist von der Kommunikationswissenschaft übernommen worden. Die Massenmedien, so geht die Auffassung heute, „konstituieren vielmehr selbst bis zu einem gewissen Grade die Realität, über die sie berichten“. So werden Ereignisse zum Zweck der Berichterstattung überhaupt erst hervorgerufen. Oder das journalistische Handeln stimuliert, beschleunigt, dramatisiert ganz bestimmte Entwicklungen der Ereigniswirklichkeit, wobei sich die daraus hervorgehenden Folgeereignisse selbst noch einmal zum Gegenstand einer Berichterstattung potenzieren können. ${ }^{T}$

In den folgenden Uberlegungen soll die Frage, wie wirklich die Fernsehwirklichkeit und überhaupt alle journalistische Medienrealität ist, auf einige philosophische und anthropologische Gesichtspunkte zugespitzt werden. Daß irgendein Wirkliches überhaupt vorhanden ist und ins Leben tritt, mit dieser Verwunderung beginnt seit alters her alle Philosophie. Es überrascht, wie selten das große Arsenal der philosophischen und kulturanthropologischen Argumente zur Erhellung des journalistischen Wirklichkeits- und Wirkungsbegriffs herangezogen wird. Journalismus, Wissenschaft und Wirklichkeitsverständnis gehen ein komplexes Bedingungsverhältnis ein. ${ }^{8}$ Journalismus wird in unseren Uberlegungen als Teil einer politischen Kultur gesehen, und hier - „Auf dem Wege zur Kommunikationsgesellschaft ${ }^{\text {“g }}$ - aktualisiert und ethisiert sich das Problem der Medienrealität über die wissenschaftliche Theorie bis in die Alltagswelt des Bürgers hinein. Neue Medien: zu welchem Ende? Der Anteil dessen, was den Menschen heute unmittelbar vor Augen tritt und persönlich erfahren wird, ist gering und scheint noch geringer werden zu sollen. Wird das Bewußtsein ein zeitungshaftes sein? Sehen wir die Welt mit Fernsehaugen?

\section{Sinnbezirk des Erkennens. Der Journalist als „Hermeneut"}

Im Fernsehen sieht Dieter Stolte die Wirklichkeit des Raumes auf zweifache Weise „vernichtet". Einmal läßt man den Raum durch die Ubertragungstechnik, die ihn überbrückt, „elektronisch verschwinden". Sodann wird dasselbe nochmals durch den zweidimensionalen Bildschirm bewirkt, wenn das räumliche Sehen durch die vom Regisseur bestimmte Perspektive abgelöst und diese Perspektive dann vom Betrachter selbst nacherzeugt wird. „Der Zuschauer verharrt unbewegt vor fremdbewegten Bildern. " So wundert sich Stolte nicht darüber, „wenn sich im Hintereinanderkonsum eines Fernsehabends, einer Fernsehwoche Sein und Schein, Fiktives und Reales, Träume und Gegenstände bis zur Unkenntlichkeit miteinander vermischen und eine eigene, wenig differenzierte Wirkmächtigkeit im Bewußtsein der Zuschauer erlangen". Es nützt auch wenig, daß die Programmgestalter ein Fernsehspiel als erfunden oder fiktiv ansagen. Das kümmert den weitaus größten Teil der Zuschauer nicht. Die Zuschauer nehmen das Flachbild zum Nennwert, „die Welt frei Haus, tagtäglich, häufig live“. Demgegenüber preist Stolte den Autor glücklich, der das Wort „Roman“ aufs 
Titelblatt schreiben und sicher sein kann, von seinen Lesern verstanden zu werden..$^{10}$ Dieses Bekenntnis des Fernsehintendanten Stolte zur "Vernichtung“ der Raum- und Wahrnehmungsrealitäten zugunsten fiktiver Strukturen könnte zu der bissigen Frage verleiten: Härte dann aller Fernsehjournalismus in Wahrheit das Wort „Roman“ auf dem Titelblatt verdient? Sicherlich nein; Stolte entzieht sich dem Dilemma auf philosophische Art, und tatsächlich kann die Antwort kaum anders ausfallen, denn sonst müßten die Fernsehredakteure ihren Anspruch auf journalistisches, d. h. realitätsorientiertes Arbeiten aufgeben und sich unter die "Dichter" einreihen. Philosophisch, das bedeutet etwa für das Genre des Fernsehspiels "Wirklichkeit und zugleich Fiktion“, womit die zutreffende Lesart für unser Thema angeschlagen wäre. Denn die Fiktion, die Phantasie, die Utopie, das seien doch wirkmächtige reale Größen, eine Wirklichkeit „auch im Kopfe", fügt Stolte mit Recht hinzu, und einmal gedacht, entstehen aus dieser Wirklichkeit wieder neue Fakten, die sich nicht allein der historischen, ökonomischen oder psychologischen Kausalität verdanken, sondern auch und vor allem gesetzter Finalität, nämlich dem menschlichen Willen. Stolte: „Die Frage ,Was ist der Mensch?' konkretisiert sich nicht zuletzt in der zukunftsgewandten Frage, Was kann ich wollen?" «11

Die Wirklichkeit (des Menschen) ebenso wie das Wollen (und Sollen des Menschen) treten hier als entscheidende Bedingungsfaktoren ins Bild journalistischer Realitätskonstruktion. Was kann ich wissen? Was soll ich tun? Die Fragen, die sich stellen, treten uns zuerst einmal als Fragen der Erkenntnissituation und der Erkenntnistheorie gegenüber; auf das Wollen und Sollen kommen wir noch zurück.

Der Sinnbezirk des Erkennens ist einer der umfassendsten und durchdringt die journalistischen Phänomene in ganz besonderer Weise. Die Information, die Nachricht, der Kommentar, die Kritik, eigentlich alles, was Massenmedien an Inhalten transportieren, ist erkenntnisfundiert. Ein Blick auf das „Wortfeld“ von Erkennen charakterisiert dieses menschliche Vermögen nach seiner Breite und Tiefe folgendermaßen: „Kennen (mit den etymologischen Zusammenhängen, die u. a. sogar in den Bereich des Geschlechtlichen hineinreichen - vgl. ,Baum der Erkenntnis') mit seinen Ableitungen und Zusammensetzungen wie Erkennen, Erkenntnis, Bekennen, Bekenntnis, Bekanntheit - einschließlich der Negationen -; weiterhin die Wortgruppe des Wissens, das sich von einer idg. Wurzel, vid' $=$ sehen (lat. videre, griech. Idea, skrt. Veden) ableitet, mit seinen Zusammensetzungen wie etwa Einsicht (lat. E-videnz); weiterhin Wortgruppen, die der Handwerkssphäre entwachsen, wie Erfassen, Be-greifen (vgl. lat. con-cipere) oder Verstehen (Ver-stand), Deuten usw. Sie bezeichnen Momente des Erkenntnisfeldes bzw. -phänomens, das in der Erkenntnistheorie philosophisch erforscht wird." 12

Aus der Etymologie und Begriffsbildung wird schon ersichtlich, daß unserer Frage nach der Wirklichkeit des Journalismus mit dem Wort- und Sinnbezirk des Erkennens ein umfassendes Programm vorgestellt wird. Es ist von philosophischer, nicht von empirisch-analytischer Art. Es ist kein wissenschaftliches Programm mit exakter Methodologie zur Objektivierung der journalistischen Wirklichkeitskonstruktion, sondern ein wertbesetztes, normatives und subjektives Programm des Erkennens und der Erkenntnistheorie. Wir bezeichneten den Journalismus bereits als Teil unserer politischen Kultur, und in diesem Bezirk, wo das Ethos der Normen und Werte ausschlaggebend ist, wird das Erkennen im Sinne des Verstehens genommen. Die Erkenntnistheorie ist hier die Hermeneutik als eine Lehre solchen Verstehens. ${ }^{13}$ Das macht den Journalismus im philosophischen Sinn zu einem hermeneutischen Phänomen. 
Den Journalisten bewegt es tagtäglich, wie Wirkliches von Unwirklichem, Reales von Erfundenem, Wissen von Scheinwissen unterschieden werden kann. Er ist der „Hermeneut" unserer Massen- und Medienkultur. Er übersetzt fortwährend den „Text" unserer Wirklichkeit in die journalistische Sprache, um ihn für die vielen Leser, Hörer und Zuschauer aufzuschließen. Das Unverständliche soll der Journalist verständlich machen, der Information und Orientierung wegen, und wie der im kultischen Vollzug handelnde "Hermeneut" der griechischen Eleusis-Mysterien einstmals der versammelten Gemeinde die Zeichenhandlungen entschlüsselte, wird heute den Massenmedien die Kunst des Verstehens und des Verständlichmachens abverlangt. Die journalistische Kunstlehre wäre dann eine Lehre von der Wirklichkeit, wie sie zu erkennen und wie sie zu deuten ist. Ihr hätte sich eine Theorie des Verstehens zuzugesellen, damit die journalistische Praxis ihr Ziel erreicht und von den Folgen für die Gesellschaft - wir sprachen von politischer Kultur und bezeichnen damit ein normatives Wertgefüge nicht absieht. Kommunikation, Massen-Kommunikation ist ein Handeln mit dem Ziel der Verständigung. Dies ist kein wissenschaftliches, sondern ein ethisches und „politisches" Ziel, ein hohes Gut, das dem allgemeinen Besten dienen soll, und darin unterscheidet sich journalistisches Erkennen und Handeln grundsätzlich von wissenschaftlichem Erkennen und Handeln; letzteres reklamiert für sich ethische Indifferenz und schon gar keine "Wahrheit". Die Idee der Wahrheit ist beherrschend im Journalismus. Wem wäre an einem „unwahren“ Erkennen gelegen? Was wirklich ist, das soll wahr sein und darüber soll der Journalist „wahrhaftig “ unterrichten, wie es ihm in Statuten und allen möglichen Programmrichtlinien abverlangt wird. ${ }^{14}$ Erich Straßner spricht von einem Berichten nach der Maxime „Sei aufrichtig!“ und räumt damit dem Wahrheits- und Wahrhaftigkeitspostulat als einem journalistischen Imperativ den gebiihrenden Rang ein. ${ }^{15}$

\section{Das Wissen vom Wissen des Journalismus. \\ Weites Verständnis von Medienwirkungen}

Was ist solchen Hinweisen zu entnehmen? Wir suchen dem Wissen vom Wissen des Journalismus näherzutreten, wie es in die Wirklichkeit (des Menschen) hineinverwoben ist und zur Bedingung aller journalistischen Wirklichkeitskonstruktion wird. Bei Platon ist die Philosophie als "Wissen vom Wissen“ gekennzeichnet. Sokrates übte sich in der Widerlegungskunst, seine Dialogpartner daraufhin zu prüfen, ob sie nur ein Scheinwissen wie die Sophisten verbreiten, oder ob sie ein fundiertes, in der Wahrheit des Wirklichen gegründetes Wissen ausweisen. Müssen wir an die Massenmedien gewissermaßen die sokratische Wahrheitssonde anlegen, ob sie als unsere Gesprächspartner nur Scheinwissen und fragmentarische Wirklichkeit vermitteln anstelle eines "Wissens vom Wissen"? Der Journalismus ist keine Philosophie. Gleichwohl kann die sokratisch-philosophische „Ars interrogandi“ für den Journalismus eine gewinnbringende Sache sein, sich des eigenen Wissens vom Wissen - wenigstens gelegentlich und paradigmatisch - zu vergewissern und sich in der "Widerlegungskunst" $\mathrm{zu}$ üben. Wir hätten das Modell eines fragend-offenen Journalismus vor uns, der die Voraussetzungen der eigenen Wirklichkeitskonstruktion zu bedenken sucht. Das Fragen, so lesen wir bei Heidegger, „ist die Frömmigkeit des Denkens“16. Aber wozu denn überhaupt Philosophie im Hinblick auf den Journalismus und seine Phänomene?

In einer von der Aufklärung bestimmten Welt kommt dem Wissen über die Welt eine große Bedeutung zu. Der Journalismus verbreitet dieses Wissen tagtäglich und legiti- 
miert sich aus dieser Wissensverbreitung. Es soll gezeigt werden, wie die Welt wirklich ist und wie das Wissen zu einem praktischen gemacht werden kann.

Zu sagen, Journalismus sei Aufklärung, setzt einen hohen Erkenntnis- und Wissensanspruch voraus, dem wir auch in der Philosophie begegnen. In Verbindung mit dem hermeneutischen Verfahren geht es auch der Philosophie um "richtiges“ Wissen über die Welt im ganzen. Die Philosophie erschließt jenes Wissen von der Wirklichkeit, das wir als Bedingung der Möglichkeit von Wissenschaft überhaupt in allen theoretischen Orientierungen wie Beobachten, Experimentieren, Berechnen, Vergleichen, Unterscheiden und in jeder praktischen Grundorientierung, sei sie moralisch, technisch oder politisch, „immer schon mit-wissen "17. Das heißt, wir stehen alle immer mitten in einem Wissen drin und beginnen niemals beim Nullpunkt, weder in der Philosophie noch beim Journalismus. Wer philosophisch fragt und forscht, entdeckt kaum etwas Neues, wie möglicherweise ein Naturwissenschaftler. Der Philosophie geht es um Klärung des Mit-Wissens, und sie greift dazu weit zurück in die Geschichte. Das bei den alten Griechen oder in der Neuzeit Erdachte und Erkannte ist heute noch als „früh Gedachtes, früh Geschicktes gegenwärtig "18. Von ihren Anfängen an hat die Philosophie als die Theorie des Wirklichen die jeweiligen Probleme ihrer Zeit aufgegriffen und dabei doch immer schon in der Tradition eines Wissens gestanden, das ihrer Zeit vorausliegt. Klärung solchen Mit-Wissens ist auch der Versuch, etwas „anders“ zu wissen als wir es zuvor gewußt haben, um es dann besser zu wissen. „Philosophieren ist Fragen nach dem Außer-ordentlichen." ${ }^{19}$

Bedarf es solcher Herleitungen des Denkens und Wissens, wenn wir das Wirklichkeitsverständnis des Journalismus erfragen wollen? Das Interpretieren und Auslegen von Ereigniswirklichkeiten ist das dauernde Geschäft des Journalismus. Den Wirklichkeiten soll ein Sinn, möglichst ein ganz "neuer" Sinn abgewonnen werden. Neuheit ist eine grundlegende Kategorie des journalistischen Selektionshandelns. Die Massenmedien haben auch strukturell und technologisch ganz neue Wege der Wirklichkeitserfassung mit sich gebracht, die der Menschheit bisher nicht zur Verfügung standen. Die entsprechenden Umbrüche im Erkennen und Bekennen dessen, was der einzelne, was wir alle als "Wirklichkeit" in unser Leben aufnehmen, lassen sich heute in den epochalen Auswirkungen noch nicht überblicken. Mit der Massenkommunikation sind Instrumente bereitgestellt worden, deren Wirkungen nicht neutral sind, und sie erreichen uns erst langsam in der vollen Breite ihrer Konsequenzen auch für die gesamte Gesellschaftsentwicklung.

Das Umfassende in der Frage nach der journalistischen Wirlklichkeitskonstruktion wird ersichtlich. Der Soziologe Kurt Lüscher fordert „ein weites Verständnis von Medienwirkungen“ unter dem "Einbezug des sozialen Kontextes“. Die Aufgaben und Leistungen der Massenmedien in der demokratischen Gesellschaft seien „prägnanter als bisher " herauszuarbeiten, und dies nicht zuletzt „aus dem Prestige und der Macht, die den elektronischen Massenmedien, ihrer Reichweite und ihrer Omnipotenz wegen

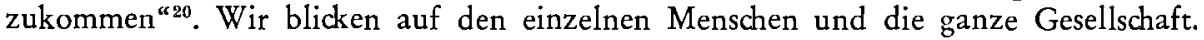
Die Massenkommunikation gibt dem einzelnen ganz neue Fähigkeiten der Wirklichkeitsbewältigung an die Hand, die von einschneidender Bedeutung für die Denk- und Lebensweise sind und deren Folgen er zu tragen hat. Auf die Dauer werden kategoriale Bestimmungen wie „Sein“, „Bewußtsein“, „Wirklichkeit“ oder „Wahrheit" über den Einfluß der Massenmedien und ihrer Methoden der Wirklichkeitserfassung auch qualitativen Veränderungen unterliegen. Das Phänomen Technik hat diese Prozesse in der Massenkommunikation ausgelöst. Daraus ergibt sich eine fortschreitende Technisierung von kategorialen Bestimmungen, die für unsere Kultur von zentralem Wert sind. 
Der Zauber dieses Phänomens zeigt sich noch immer in den faszinierenden Wirkungen, die heute insbesondere von den elektronischen Medien für große Zuschauermassen ausgehen. Otto B. Roegeles Frage „Kann die Demokratie das Fernsehen überleben?“ ist nicht nur von rhetorischer $\mathrm{Art}^{21}$; hier ist allein an die Ausübung der Thematisierungsfunktion zu denken, oder an die Verwischung der Grenzen zwischen Politik und Unterhaltung.

\section{Moralität der Selbstverpflichtung. Verantwortung und Freibeit}

Der einzelne, wir alle, ob Kommunikatoren oder Rezipienten, haben die Folgen der journalistischen Wirklichkeitskonstruktion zu tragen und $\mathrm{zu}$ verantworten, und hier ist die Rede auf das journalistische Wollen und Sollen zu bringen. Verändert der Journalismus unsere Welt? Prägt er die Anschauungen und das Denken, die Gefühle und die Werte? Bestärkt er die Vernunft in der Gesellschaft? Oder vergrößert er die Ratlosigkeit und die Orientierungslosigkeit? Vertieft er den Graben zwischen System und Freiheit? Oder ermutigt er das freiheitsfördernde Wissen vom Menschen und seiner Lebenswelt? "Verantworten heißt, für Wirkungen einstehen, die man verursacht. "22 Die Fragen, die sich im Hinblick auf die Folgewirkungen stellen, nehmen einen freien Journalismus in einer freien Gesellschaft in die Selbstverpflichtung, der allerdings die vernünftige Begründung entzogen würde, wenn ihr nicht das Erkennen vorausgeht, nach dem Wirklichen als einem Wirkenden zu forschen, um es aufzuhellen, was es ist und was es für den Menschen bedeutet.

Die Theorie der Wirklichkeitserkenntnis und die Frage nach dem Wollen und Sollen des Menschen gehören zusammen. Die Ethik als eine Sollenslehre für den Menschen ist auf dem Fundament einer Seins- und Wirklichkeitslehre gegründet; sonst wäre sie auf Sand gebaut.

Angewandt auf das journalistische Untersuchungsfeld, fragt eine Philosophie als Erkenntnistheorie nach dem Wissen vom Wissen der in der öffentlichen Kommunikation hervorgebrachten Wirklichkeit mit den ihr zukommenden Bestimmungen. Wirkliches, sofern es Wahres ist, benennt eine dieser Bestimmungen, die den Journalismus, der unter dem Anspruch einer "wahrheitsgetreuen“ Berichterstattung steht, rechenschaftspflichtig macht. Dieses Fragen kommt nicht aus dem Vermögen des Journalismus, sondern es bedarf der Reflexion und philosophischen Anschauung, den Journalismus im ganzen zu thematisieren und den Weg von der bloß richtigen Meinung zum Wissen vom Wissen des „wahren "Wirklichen zu gehen. Das Ziel ist, wenigstens in Ansätzen, eine Theorie des Journalismus, und die Methode unserer Untersuchung ist die Begründung der Moral durch Freiheit.

Die Philosophie ist nach Martin Heidegger „in ihrem innersten Grund radikalste, universalste und strengste begriffliche Erkenntnis". Der Probierstein der philosophischen Wahrheit, heißt es weiter, „liegt einzig in der Treue des einzelnen Philosophierenden zu sich selbst". Zwar müsse das Philosophieren immer durch ein strenges begriffliches Wissen hindurch und in dessen Medium bleiben, „aber dieses Wissen ist in seinem echten Gehalt nur dann begriffen, wenn in ihm zugleich die ganze Existenz in ihrer von der Philosophie gesuchten Wurzel ergriffen ist - in der Freiheit “23.

Erkenntnis und Freiheit, das sehen wir hier, gehen zusammen, und dies gilt ganz elementar auch für den Journalismus und seine Wirklichkeitskonstruktion. Von Ethik reden, das heißt, von Freiheit sprechen. Nur im freien Einverständnis, dies zu tun, 
jenes zu unterlassen, ist die journalistische Sittlichkeit zu begründen. Sonst hätten wir eine Sklavenmoral, bestenfalls eine reine Anpassungsmoral vor uns.

Der Journalismus, den wir vorfinden, ist mit der Wirklichkeit unserer realen Freiheit, die in der Demokratie als Meinungsäußerungsfreiheit nachweisbar ist, weitgehend identisch. Das ist ein empirischer Befund. Woran orientiert sich diese reale Freiheit im praktischen Handeln eines Journalisten? Der Wille des Menschen ist frei, unter mancherlei Bedingungen selbst die Ziele und Regeln für sein Handeln auszuwählen, Ordnungen in ihrer Vernünftigkeit zu begründen. Das rechte Handeln und das gute Leben - ein "geglückter" Journalismus ist hier gemeint - treffen sich in dieser handlungs-, regel- und ordnungsbegründenden Freiheit, die praktische Freiheit heißt. ${ }^{24}$

Das Ethos des Journalismus ist die praktische Freiheit. Die Moralität des Journalismus findet darin ihren Grund. Reale Freiheit, praktische Freiheit: diesen Freiheitsbegriffen wird ein dritter zugesellt, den die Philosophen transzendental nennen. Er ist Ausdruck eines Umfassenden, das die Lebenswelt des einzelnen umgreift, „Anerkennung von Freiheit durch Freiheit“25. Der Ursprung des Guten - eines guten Journalismus liegt in dieser unbedingten Anerkennung als Bedingung der Möglichkeiten eines verantwortbaren Journalismus in unserer Gesellschaft.

\section{Natur, Technik, Wissenschaft. Primäre und sekundäre Erfabrung}

Wir kehren zu der Frage zurück, welche Wirklichkeit der Journalismus hervorbringt; Ethik und Ethos des Journalismus konnten hier als Thema nur gestreift werden. Hinsichtlich der Wirklichkeitskonstruktion ist das Verhältnis von Natur und Technik zu bedenken. Strenggenommen müssen wir den Naturbegriff in Anführungszeichen setzen, weil sich Natur als das fraglos Vorhandene und Vertraute unserem Erkennen niemals darbietet, sondern der Weg dorthin zu einer quasi-ursprünglichen Weltstruktur immer schon verbaut ist. Kultur ist bearbeitete Natur. Wissenschaft und Technik sind Bearbeitungsformen des Wirklichen. Hat es jemals eine technikfreie Menschengesellschaft gegeben? Ist die Wirklichkeit, solange es den Menschen als Kulturwesen gibt, nur über die Wirklichkeitskonstruktion vermittelt worden?

Solche Fragen stellen sich im Hinblick auf die Instrumente der modernen Massenkommunikation, die sich als Werkzeuge des "homo faber" vorstellen. Können die Massenmedien nur die Brechungen der Wirklichkeit verfügbar machen, das Künstliche und journalistisch Hergestellte als ein der Natur Entgegengesetztes? Der Journalismus: Realität aus zweiter und dritter Hand?

Zur Beantwortung dieser Fragen greifen wir auf anthropologische und kulturanthropologische Erkenntnisse zurück und setzen sie in Beziehung zum Journalismus. Der Mensch ist dadurch ausgezeichnet, daß seine Natur „von Freiheit ist", wie Jörg Splett ausführt. „Das Wesen, die Wirklichkeit, zu dem der Mensch hervorgeht, ist kein im vornhinein bestimmtes und festliegendes, sondern in seinem Hervorgang bestimmt er selbst erst über die Wirklichkeit, zu der er wird. "28 Man könnte es auch anders ausdrücken und formulieren, der Mensch sei das Wesen des distanzierenden Denkens und Erkennens, der das in der Natur zu Tage Getretene nicht unmittelbar, sondern im Gegen-Stand wahrnimmt. "Wirklich“ ist für ihn, was er sich vergegenständlichen kann und somit dem erkennenden Zugriff unterbreitet wird. „Wirklich“ ist, was er sich vorstellen kann. „Vor-stellen bedeutet hier: das Vorhandene als ein Entgegenstehendes vor sich bringen, auf sich, den Vorstellenden $\mathrm{zu}$, beziehen und in diesen Bezug zu sich als den maßgebenden Bereich zurückzwingen. “27 
In der Kommunikationswissenschaft ist die systemtheoretische Auffassung, daß jedwelche Wirklichkeit als Reduktion komplexer Ereignishaftigkeit von Welt begriffen wird, zur Grundvoraussetzung für journalistisches Handeln erklärt worden. Wissenschaft und Journalismus, die Manfred Rühl eng zusammenrückt, „sehen sich nie einer Nullsituation, sondern stets einer durch den Alltagsverstand vortypisierten Wirklichkeit konfrontiert". Diese Alltagswirklichkeit werde von der, mit Methoden- und Theoriebeständen gerüsteten Wissenschaft grundsätzlich problematisiert und selektiert; aufgrund der Ergebnisse des Forschungsprozesses werden sie „als eine andere Wirklichkeit konstruiert " ${ }^{\text {28 }}$. Hier wird der Journalismus über die Wissenschaft hergestellt, aber deren Objektivitätskriterien stehen diametral zu den journalistischen Geltungsgründen, die von moralischer und nicht wissenschaftlicher Art sind; entsprechend greift die Systemtheorie viel zu kurz, wenn sie nur reduktive Mechanismen, Funktionen und Prozesse bei der journalistischen Wirklichkeitskonstruktion am Werk sieht, mögen diese auch als "hochkomplex" geschildert werden. Außerdem nährt sie die trügerische Hoffnung, der Journalismus könnte „evolutionär" sich doch einer quasiwissenschaftlichen Objektivität annähern und aus der Logik seiner Systemrationalität die moralischen Entscheidungs- und Sinnstrukturen als „vorwissenschaftlich" zurückweisen. Verantwortung und Freiheit sind in dem systemtheoretischen Kontext schwerlich unterzubringen. Die Ideen und das Geistige, die das spezifische Humane im Journalismus konstituieren, werden zu Randerscheinungen.

Es gilt aber gerade, den Journalismus vom Menschen her zu denken und ihn seinen Zwecken dienstbar zu machen; die anthropologische und geisteswissenschaftliche Sichtweise ist unter solchen Aspekten viel ergiebiger. Seit es eine Menschheit gibt, hat der Mensch seine eigene Natur organisiert und ist die Wirklichkeit, die der Mensch erkennend wahrnimmt, kulturell geprägt. So wird das Vorhandene zum Vertrauten, das als ein wirkend Wirkliches ins Leben eintritt.

Wenn wir die Natur eine primäre Welt nennen wollen, entfernt sich der Mensch dauernd von ihr in die sekundäre Welt seiner Kultur- und Wirklichkeitserfassung. Sekundärerfahrung, deren Vermittlung den Massenmedien oft in kulturpessimistischer Beurteilung vorgehalten und vorgeworfen wird, wäre dann eine kulturell geprägte Erfahrung, deren Abwertung gegenüber der sogenannten Primärerfahrung grundlos erscheint. Die Technik eines Mediums tritt dazwischen. Die Botschaft fließt durch den Kanal. In einem gewissen Sinne "ist" das Medium die Botschaft. ${ }^{29}$ Der Weltstoff wird gefiltert und zur "Information“ verarbeitet.

\section{Maschinenwelt und Massenkommunikation. \\ Kulturantbropologische Wirklichkeitserfabrung}

Mit der Neuzeit und ihren technischen Errungenschaften hat sich die Wirklichkeitsbearbeitung des "homo faber" potenziert. Die in seiner Vorstellung verfügbar gemachte Wirklichkeit kann er nun vollends in den Griff nehmen. Er beherrscht die Naturkräfte, wie wir sagen. Lewis Mumford betrachtet die moderne Technik nicht als völlig neues Phänomen, das seinen Ursprung in der sogenannten industriellen Revolution des achtzehnten Jahrhunderts hat, sondern er glaubt sie schon "ganz am Anfang in der Organisation einer archetypischen Maschine, die aus menschlichen Teilen bestand", nachweisen zu können. Mumford bezeichnet den Körper des Menschen als Allzweckwerkzeug. Der Mensch sei nur mittels der Kultur imstande, seine eigene Natur auszuschöpfen, kontrollieren und voll entwickeln zu können. Heute haben wir genügend 
historische Perspektive gewonnen, um erkennen zu können, daß dieser scheinbar vollautomatisierte Mechanismus unserer Maschinen „in seinem Getriebe einen Menschen versteckt hat; wir wissen, daß das System nicht direkt aus der Natur abgeleitet ist, wie wir sie auf der Erde oder im Himmel vorfinden, sondern Züge aufweist, die an jedem Punkt den Stempel des menschlichen Geistes tragen, teils rational, teils schwachsinnig, teils dämonisch" 30 .

Dieses Statement ist voll übertragbar auf die Maschinenwelt der Massenkommunikation; im Getriebe des journalistischen Systems hat sie „einen Menschen versteckt“, und über einen Mangel an "Schwachsinn" in den Massenmedien brauchen wir uns nicht zu beklagen. Die Angst unseres Zeitalters ist es gerade, der Mensch möchte zum Zauberlehrling werden, der am Ende die Formel vergißt und unter die Herrschaft der Apparate gerät, wenn die Technik aus dem Lebenswelthorizont isoliert wird und der „homo faber" zur Machtergreifung über den "homo sapiens" schreitet.

Wenn wir die Technik kulturanthropologisch betrachten, stellt sie sich als Prozeß der Entwirklichung dar. Das ursprünglich Zukommende geht in die Vergegenständlichung ein und wird damit zugedeckt. Die Entwicklungslinie verläuft von der Erzeugung des Feuers und der Erfindung des Pflugs bis zu jenen Zwischen- und Gegenwelten, die wissenschaftlich-technisch hergestellt werden, wie die journalistischen "Gegenwelten" zum Beispiel.

Der Mensch tritt mit seinen Bauplänen an die Wirklichkeit heran und schöpft aus ihr, die Welt zu vergegenständlichen und sie sich durch diese Objektivierung zu eigen zu machen. Diese seine Freiheit bleibt jedoch an das Erkennen gebunden. Die dem Erkennen gestellte Aufgabe wird von Erich Rothacker, der vom Kultusbegriff her philosophiert, als das "Eindringen in die Struktur dieser einen Welt als wahrer Wirklichkeit" gekennzeichnet. Der Mensch erdeutet sich schöpferisch seine Welt, und dabei nimmt der Kulturanthropologe eine strenge Korrelation von Bauplan und erlebtem Wirklichkeitsinhalt gewahr. Doch gleichzeitig erinnert er daran, wie dem menschlichen Erkennen „eine dunkle, rätselhafte, extensiv und vor allen Dingen intensiv vollkommen unausschöpfbare Wirklichkeit" gegenübertritt. Sehr schön fügt Rothacker hinzu: „Denn jedes Stückchen dieser Wirklichkeit ist von unendlicher intensiver Mannigfaltigkeit. Es ist restlos nie ausschöpfbar. Nichts ist intensiv restlos ausschöpfbar für unsere Erkenntnis. Die Erkenntnis bleibt hier immer im Rückstand. Wir sind also zu einer Selektion gezwungen, und diese Auslese des Bedeutsamen ist zunächst einmal auf der Folie der unausschöpfbaren Wirklichkeit ärmer. " ${ }^{\text {31 }}$

So ist das Erkennen (und sicherlich das journalistische Erkennen) ein begrenztes Stückwerk und die ihm zu Hilfe kommende Technik ein Flickwerk, weil die Wirklichkeit (die journalistische Ereigniswirklichkeit) zu komplex ist, vom menschlichen Bewußtsein in ihrer unbegrenzten Fülle aufgefaßt zu werden. Zwar kann der Mensch im Unterschied zum Tier die ganze Wirklichkeit auf die Idee bringen, auf die Idee der einen Welt, wie sie ihm erscheint, aber damit hat er noch nicht den Graben überbrückt, „die Idee eines Unterschiedes dieser einen Welt als eines Inbegriffs von Dingen an sich im Unterschied zu Erscheinungen, in denen sie sich ihm bietet, des einen Seins gegenüber der Mannigfaltigkeit des Seienden. Kurz: er ist von vorneherein auf dem Weg zur Philosophie und zur Wissenschaft ${ }^{\text {" } 32}$. Das heißt, der Mensch ist einerseits in die kulturelle Umwelt seiner spezifischen Wirklichkeitserfahrung eingebunden. Andererseits hat er Distanz zur Wirklichkeit und besitzt Weltoffenheit. Er kann seinen jeweiligen Horizont überschreiten, und der Mensch "philosophiert" unausgesprochenermaßen bereits, indem er dies tut. 


\section{Medienrealität und Nadbrichten. Objektivität als Annäherungswert}

Die Kommunikationswissenschaft nähert sich dem Problem, welche Wirklichkeit der Journalismus hervorbringt, anders als die Philosophie, aber auch sie gelangt zu dem Ergebnis einer "sekundären" Realität. So hat man die Medienrealität der Nachrichten untersucht, um herauszufinden, daß die von den Medien transportierte Realität mit der tatsächlichen Ereigniswirklichkeit nicht übereinstimmt. Dieser Falsifikationsansatz sollte zeigen, daß die Instrumente der sozialen Kommunikation aufgrund eigener Strukturen und Arbeitsgewohnheiten das wirkliche Geschehen nicht reproduzieren können. Ein solcher Forschungsansatz läuft letzten Endes immer auf den Vergleich hinaus, die von den Medien vermittelte Realität der „faktischen“ Realität gegenüberzustellen und jene an dieser zu messen. Diesen Ansatz hat Winfried Schulz in seiner Analyse der aktuellen Berichterstattung mit Recht verworfen. Die Frage, ob das Bild, das die Medien von der Realität zeichnen, „richtig" ist, läßt sich nach der Auffassung dieses Medienforschers mit den Mitteln der Wissenschaft nicht beantworten. Was "wirklich" geschah, welches das "richtige“ Bild von Realität ist, das sei eine letztlich metaphysische Frage, und niemand sei in der Lage, darüber eine intersubjektiv verbindliche Auskunft zu erteilen. ${ }^{39}$

Müssen wir also das Problem an den Philosophen weitergeben? Jeanne Hersch hat einmal bemerkt, der Philosoph befasse sich mit unlösbaren Problemen; sobald sich nämlich eine Lösungsmöglichkeit ergibt, würden sie zu wissenschaftlichen Problemen. ${ }^{34}$ Der Philosoph weiß das "richtige“ Bild von der Realität ebensowenig zu bestimmen wie der Kommunikationsforscher. Wohl aber kann er fragen: Wenn Nachrichten nicht „wahr" sind, was sind sie dann? Erfundene Nachrichten will doch niemand hören und sehen. Die Nachrichten werden von denen, die sie aufnehmen, als "wirklich“ erfahren, und dies nicht deshalb, weil ihre „Richtigkeit" wissenschaftlich erhärtet werden kann, sondern weil im Journalismus das Aufrichtigkeits- und Wahrheitspostulat gilt - gelten soll. Nachrichten müssen "stimmen". Nachrichten sind wahre Nachrichten; sonst sind sie Schein, Täuschung, Irrtum, Betrug, Dummheit. Auf den Annäherungswert, den wir journalistische Objektivität nennen, kommt es an.

$\mathrm{Zu}$ den Grundmustern, nach denen im Journalismus die Selektion, Interpretation und Sinngebung von Realität erfolgt, müssen Redlichkeit und Sachlichkeit treten, den Journalismus als ein "bonum" zu realisieren. Diese journalistische Moral wird weithin nicht reflektiert; sie ist eine gelebte Moral und bewährten Handwerks- oder Kunstregeln vergleichbar, ein gutes Werk, einen guten Journalismus zu erbringen. Nichtsdestoweniger sind es ethische Qualitäten, die ins Bild treten und entscheidend sind, nach welchen Werten und Faktoren etwa eine Nachrichtenwirklichkeit zu „organisieren “ ist. „Man kann also sagen, daß Nachrichten ,Realität" eigentlich konstituieren“, folgert Winfried Schulz. ${ }^{35}$

Das Wissen darüber, daß die Medien ihre eigene Realität konstruieren, ist offenkundig. Doch es ändert, wie wir bereits eingangs bemerkten, wenig daran, daß die Menschen im Regelfall die Medienrealitäten als verbürgte Zeugnisse eines tatsächlichen Geschehens aufnehmen. Der Mensch scheint andauernd auf der Suche nach Realität zu sein; er will sich in ihr wiedererkennen und überhaupt erkennen. Was er in Wahrheit hat, sind Erfahrungen und Geschichten, die das eigene Leben lesbar machen. Erzählen die Medien vielleicht überhaupt nur "Geschichten“? Wir wollen die Zweifel nicht zu weit treiben, aber die Doppelbödigkeit des journalistisch Authentischen sollte gegenwärtig sein. Die Erfahrung "dichtet" und mittels der Fiktion "erfahren" wir das Leben, fremdes und eigenes. 
Auch insofern ist die Unterscheidung zwischen Primär- und Sekundärerfahrung im Medienbereich wenig hilfreich. Die meisten Zeitgenossen sind nicht so naiv, die Zeitungs- oder Bildschirmrealität mit jenen Wirklichkeiten zu verwechseln, die sie tagtäglich im persönlichen Umgang mit ihresgleichen erleben. Die Wirkungen, die ausgelöst werden, scheinen jedoch auf die unterschiedlichen Erfahrungsquellen wenig Rücksicht zu nehmen. Nicht nur für Kinder, sondern auch für Erwachsene kann ein Fernsehprogramm in höchstem Maße erlebtes Leben bedeuten, mögen die Erfahrungen auch nur aus zweiter Hand stammen. Der Mensch macht sich ein Bild von vielen Dingen, die der unmittelbaren Sinneswahrnehmung nicht zugänglich sind, und seine Reisen ins Land Utopia nehmen kein Ende.

\section{Journalismus als moralische Wirklichkeit. Anstrengung der ethischen Vernunft}

Das ethische Problem heute ist das Auseinanderdriften von Technik und Humanität. Wir fragten nach den neuen Medien: zu welchem Ende? In den letzten Jahren ist die öffentliche Sensibilität dafür gewachsen, daß die Herauslösung der Technik aus der Moral als eine Fehlform menschlichen Existierens und politischer Kultur anzusehen ist. Die Massenmedien in der modernen Welt sind ein Teil jener globalen „Stadt“, von der Hans Jonas spricht, der in seinem Buch „Das Prinzip Verantwortung“ eine Ethik für die technologische Zivilisation entwirft. ${ }^{36}$ Dieser Autor sieht die Stadt der Menschen als das totale Artefakt, als die Summe der zur Welt gewordenen Werke des Menschen, die auf ihn und durch ihn selbst wirken, über das Ganze der irdischen Natur ausgebreitet, deren Platz die Technologie usurpiert hat. Der Unterschied zwischen dem Künstlichen und dem Natürlichen sei verschwunden, das Natürliche sei von der Sphäre des Künstlichen verschlungen worden, und die menschliche Freiheit sehe sich vor eine neue Art von „Natur“ gestellt. ${ }^{37}$

Die Wirklichkeit, die der Journalismus mittels seiner Kommunikationstechnologien in der globalen "Stadt ${ }^{*}$ hervorbringt, ist eine moralische Wirklichkeit, die unter dem Postulat der Freiheit steht, unser Können im Horizont des Erkennens zu halten. Wir können die Technik des Menschen nicht aus der sie begründenden Vernünftigkeit entlassen, sondern müssen dafür Sorge tragen, daß alle Richtigkeit sich durch die Frage nach ihrer Moralität legitimiert.

So scheint die journalistische Wirklichkeit, die von den Medien hervorgebracht wird, wie kaum eine andere heute philosophiebedürftig $\mathrm{zu}$ sein. "Journalismus", schreibt Ulrich Paetzold, „muß in einer Demokratie eine praktische Philosophie haben. ${ }^{\text {“38 }}$ Der Journalismus darf sich nicht der Anstrengung des ethischen Denkens und der ethischen Vernunft entziehen. Die Technisierung unserer Lebenswelten, die heute - zum Ende des Gutenberg-Zeitalters? - die Menschen auch über die Massenmedien und die Elektronik mit voller Wucht erreicht, ist ohne den Rückbezug auf das Wahre und Gute, wodurch eine bloß funktionierende Technik ins Unrecht gesetzt werden kann, auf dem Weg in eine selbstzerstörerische Barbarei.

Das entsprechende Forschungs- und Untersuchungsfeld ist für den Journalismus noch kaum ausgemessen worden. Wie in der Massenkommunikation das Wirkliche als das Wirkende und Wirksame produziert wird und unser menschliches Bewußtsein von der Welt, in der wir leben, davon geprägt ist, das öffnet ein weites und interdisziplinäres Feld, wo Alltagspraxis und Wissenschaft, Sinneswahrnehmung und Erkennen, Wirklichkeits- und Wahrheitstheorie miteinander in Beziehung treten. 
Die Philosophie blickt seit ihren Anfängen auf die vorwissenschaftliche Erfahrung des In-der-Welt-Seins, daß der Mensch immer schon (die Wirklichkeit) erfahrend in der Welt ist und ihr doch nicht angehört wie das Tier, das ganz im Konkreten und Wirklichen lebt. Max Scheler zitiert Buddha mit einem Ausspruch — „herrlich sei es, jedes Ding zu schauen, furchtbar es zu sein" - und knüpft daran eine Definition des Menschen, der aller Wirklichkeit in ihrem Jetzt und Hier ein kräftiges "Nein" entgegenschleudert. Der Mensch wird als „Asket des Lebens“ geschildert, der kraft seines Geistes diesen Akt der Entwirklichung vollzieht, der „ewige Protestant gegen alle bloße Wirklichkeit ... die Schranken seines Jetzt-Hier-Soseins zu durchbrechen, immer strebend, die Wirklichkeit, die ihn umgibt, zu transzendieren "39. Scheler fügt eine Bemerkung hinzu, die uns sofort an den Sensations- und Neuigkeitshunger der Massenmedien denken läßt, der etwas Unersättliches hat. Er nennt den Menschen im Verhältnis zum Tier, dessen Dasein "das verkörperte Philisterium" sei, den ewigen Faust, die „bestia cupidissima rerum novarum ${ }^{440}$.

Letzten Endes wirft die journalistische Neugier den Menschen auf sich selbst zurück. Er sieht dauernd sein eigenes Bild. Novalis sagt zu dem Mann, der wagemutig den Schleier vom Antlitz der Göttin Isis wegzieht: „Und was sah er? Er sah, Wunder der Wunder, sich selbst!"

Die Medienrealität ist ein Artefakt des Menschen, wie wir gesehen haben, und die Massenmedien sind ein Stück unserer politischen Kultur. Sie bedürfen der Einbindung in die Wertverhältnisse, die unsere „Umwelt" sind und deren hervorstechendes Merkmal der anthropologische Rückbezug ist: Würde des Menschen. In den letzten Jahren hat die öffentliche Aufmerksamkeit für die ethischen Probleme generell zugenommen; die Grundwertdebatte ist ein Ausdruck dieses Trends. In vielen Bereichen wird die Ethik zur Uberlebensfrage, wenn wir an die Natur- und Umweltzerstörung denken oder Fragen der Friedenssidherung herausgreifen. Im Journalismus, so könnte manch einer denken, liegen die Dinge nicht so dramatisch. Das ist ein Irrtum. Ohne einen freien und funktionierenden Journalismus ist die demokratische Lebensform nicht $\mathrm{zu}$ erhalten. Der Journalismus ist konstitutiv für sie, das gemeine Beste in der öffentlichen Debatte immer aufs neue zu artikulieren. Die ethischen Fragen stellen sich nicht nur im individuellen Alltag derer, die den Journalismus produzieren und rezipieren, sondern ebenso aus der Perspektive der gesellschaftlichen Vernunft, was der Journalismus im ganzen unserer Gesellschaft bewirken soll, für wen hier eigentlich gearbeitet wird und inwieweit die Leistungen der Massenkommunikation dem guten Zweck genügen, für den sie sich erklären und zu bürgen haben.

\section{Anmerkungen}

1 Vgl. Paul Watzlawik (Hrsg.): Die erfundene Wirklichkeit. Wie wissen wir, was wir zu wissen glauben? Beiträge zum Konstruktivismus, München 1981.

2 Drawing by Robert Mankoff; reprinted from the Saturday Review. Vgl. Elisabeth NoelleNeumann: Die Schweigespirale. Offentliche Meinung - unsere soziale Haut, München $1980,216$.

3 Werner Heß anläßlich des 25jährigen Bestehens des ARD-Programmbeirats. Heß, ausgeschiedener Intendant des Hessischen Rundfunks, meinte zum Verhältnis von Fernsehen und Realität, daß niemand mehr in der Lage sei, von sich aus zu klären, ob die vom Medium ausgebreiteten Signale der Wirklichkeit entsprechen. - Werner Heß: Der mündige Bürger wird immer mehr zum manipulierten Bürger, in: Frankfurter Allgemeine Zeitung, 1.4.1981. 
${ }^{4}$ Dazu Winfried Schulz: Die Konstruktion von Realität in den Nachrichtenmedien. Analyse der aktuellen Berichterstattung, Freiburg 1976, 29.

5 Peter L. Berger, Thomas Ludkmann: Die gesellschaftliche Konstruktion der Wirklichkeit. Eine Theorie der Wissenssoziologie, Frankfurt 1974, 21.

- Vgl. Walter Lippmann: Public Opinion, First Free Press Paperback Edition, New York 1965, Copyright 1922.

7 Hans Mathias Kepplinger: Die Grenzen des Wirkungsbegriffes, in: Publizistik $1-2 / 1982$, 108. Walter Flemmer, Leiter der Programmgruppe Erziehung und Bildung im Bayerischen Rundfunk, trug auf den Hohenheimer Medientagen 1981 das folgende Beispiel vor: „Vor wenigen Wochen protestierten die Mieter eines Schwabinger Wohnhauses gegen die Absicht, ihr Haus modernisieren zu lassen, dadurch, daß sie sich, Fenster für Fenster, zur Straßenfront hin mit nackten Hintern zeigten. Bei diesem Akt, bei diesen Rückenakten war das lokale Boulevardblatt dabei und brachte das Foto der Aktion groß an hervorragender Stelle. Man muß sich fragen: Hätten die Leute ihre Hinterteile in die Fenster gehängt, wenn die Presse nicht dabei gewesen wäre?" Natürlich nicht; es ging ihnen um Publizität und "Herstellung" von Offentlichkeit. Flemmer berichtete weiter, wie im Anschluß daran ein Gymnasium für den Sozialkundeunterricht Schüler in dieses Schwabinger Mietshaus schickte, um sie als Journalisten agieren zu lassen. Die Schüler sollten Interviews mit den Mietern machen, Protokolle anfertigen, Stellung beziehen zu der geplanten „Kaputtsanierung“. Flemmer fuhr fort: „Wenn nun das Fernsehen dies wiederum zum Anlaß nähme, um über den wirklichkeitsnahen Unterricht eines fortschrittlichen Lehrers zu berichten, stünden wir mitten drin im Kreis einer möglicherweise für die Publizität erzeugten Wirklichkeit." Walter Flemmer, Realität im Fernsehen? in: Ethik und Kommunikation, Hohenheimer Medientage 1981, $20 \mathrm{ff}$.

s Vgl. zu diesem Bedingungsverhältnis den Aufsatz von Manfred Rühl: Journalismus und Wissenschaft - Anmerkungen zu ihrem Wirklichkeitsverständnis, in: Rundfunk und Fernsehen 1981/2-3, $211 \mathrm{ff}$.

9 Unter dieser Öberschrift brachte die Fachzeitschrift „Publizistik“, Heft 4/1982, 481, die Beiträge zum Kolloquium des Burda-Preises für Kommunikationsforschung nin MEDIAS res".

10 Dieter Stolte: Die Phantasie als Schlüssel zur Wirklichkeit, in: Fernseh-Kritik. Wirklichkeit und Fiktion im Fernsehspiel, hrsg. von Anna-Luise Heygster und Dieter Stolte, Mainzer Tage der Fernseh-Kritik, Band XI, Mainz 1980, 7.

11 Ibid., 9 f.

12 Alwin Diemer, Ivo Frenzel (Hrsg.) Fischer-Lexikon Philosophie: Frankfurt 1980, 32.

13 Ibid., 39.

14 Vgl. Hermann Boventer: Der Journalist und die Pilatusfrage, in: Kurt Koszyk, Volker Schulze (Hrsg.), Die Zeitung als Persönlichkeit, Festschrift für Karl Bringmann, Düsseldorf 1982, 29-40.

15 Erich Straßner: Fernsehnachrichten. Eine Produktions-, Produkt- und Rezeptionsanalyse, Tübingen 1982, 82. Straßner bringt das Aufrichtigkeitspostulat im Zusammenhang mit drei weiteren Imperativen zum Berichten jeweils als ein Handeln nach der Maxime „Sei verständlich!“, „Sei informativ!“" und „Sei relevant!“. Er entwickelt daraus eine kleine Ethik für den Nachrichtenredakteur, untergliedert in kommunikationswissenschaftliche Vorgaben und redaktionelle Praxis.

10 Martin Heidegger: Vorträge und Aufsätze, Pfullingen 1954, 44.

17 Manfred Riedel: Philosophieren nach dem Ende der Philosophie? Zur Sache des Denkens im Zeitalter der Wissenschaft, in: Wozu Philosophie? Stellungnahmen eines Arbeitskreises, hrsg. von Hermann Lübbe, Berlin 1978, 278.

18 Martin Heidegger, a. a. O., 48.

19 Martin Heidegger: Einführung in die Metaphysik, Tübingen ${ }^{2} 1958,10$.

20 Kurt Lüscher: Medienwirkungen und Gesellschaftsentwicklung, in: Media-Perspektiven 9/1982, 545, 552.

21 Vgl. Otto B. Roegele: Neugier als Laster und Tugend, Zürich 1982, 42 ff. 
${ }^{22}$ Hans Sachsse: Technik und Verantwortung. Probleme der Ethik im technischen Zeitalter, Freiburg 1972, 8.

${ }^{23}$ Martin Heidegger: Ges. Ausgabe Bd. 26. Metaphysische Anfangsgründe der Logik im Ausgang von Leibniz, Frankfurt 1978, 22.

24 Vgl. Hermann Krings: System und Freiheit, München 1980, $40 \mathrm{ff}$.

${ }^{25}$ Ibid., 68.

${ }^{26}$ Jörg Splett: Natur. In: Sacramentum Mundi, III. Band, Freiburg 1969, 675 f.

27 Ibid., 676.

${ }^{28}$ Manfred Rühl, a. a. O., 220.

${ }^{28}$ Dazu Hermann Boventer: Der elektronische Mensch, Medium und Botschaft. Szenenwechsel für Marshall McLuhan? in: Communicatio Socialis, 1/1982, $37 \mathrm{ff}$.

${ }^{80}$ Lewis Mumford: Mythos der Maschine. Kultur, Technik und Macht, Frankfurt 1977, 23, 833.

${ }^{31}$ Erich Rothacker: Philosophische Anthropologie, Bonn 21966, 86, 82. Rothackers Frage nach der Wirklichkeit aus kulturanthropologischer "Wirklichkeitsdefinition" hat Rainald Merkert in einem lesenswerten Aufsatz „Fernsehen und Wirklichkeit", in: Stimmen der Zeit, 7/1982, 483-495, für die Fernsehwirklichkeit thematisiert.

${ }^{32}$ Erich Rothacker, a. a. O., 86.

${ }^{33}$ Winfried Schulz, a. a. O., 27.

34 Jeanne Hersch: Die Unfähigkeit, Freiheit zu ertragen, Zürich 1974, 7.

35 Winfried Schulz, a. a. O., 28.

${ }^{36}$ Hans Jonas: Das Prinzip Verantwortung. Versuch einer Ethik für die technologische Zivilisation, Frankfurt 1979, 33.

37 Ibid., 34.

38 Ulrich Paetzold: Hofberichterstattung oder Recherchen-Journalismus. Zur Philosophie journalistischer Arbeit, in: Wolfgang R. Langenbucher (Hrsg.): Journalismus \& Journalismus. Plädoyers für Recherche und Zivilcourage, München 1980, 30.

39. Max Scheler: Die Stellung des Menschen im Kosmos, Bern ${ }^{6} 1962,52,55 \mathrm{f}$.

${ }^{40}$ Ibid., 52.

\section{SUMMARY}

Walter Lippmanns "Pictures in our Heads" have been part of an explanation of media reality. But how in fact does this reality look! How real is especially the television with its influence on the values of society? Here the author matches philosophical considerations and those of social anthropology with journalism. Thus the whole area of recognition permits communications work in a very specific way. Hermeneutics and journalism must meet. At the same time one sees how a theory of recognition of reality demands an ethic of journalism. The ethical dimension is also shown in the divergence between techniques and human life. The technical instruments of journalism cannot be released from reasonable intelligence. All sincere journalism legitimates itself through its ethics.

The philosophies of truth and of freedom point to a necessary journalistic reality. Journalism demands a practical philosophy.

\section{RÉSUME}

Le livre "picture in our head" par Walter Lippmann, était une tentative d'explication sur la problématique de la construction de la réalité journalistique. Mais comment se présente effectivement la réalité des média et notamment la réalité télévisive avec son influence sur le comportement estimatif de la population? L'auteur présente ici des considérations philosophiques et anthropologiques les approchant au journalisme. Notamment, le sens recognitif est 
inhérant dans le journalisme. L'herméneutique et le journalisme se joignent. En même temps, il est démontré comment une théorie de définition de la réalité journalistique provoque une éthique du journalisme. Le problème éthique s'avère dans le journalisme comme séparation de la technique et de l'humanité. L'instrument technique du journalisme ne peut pas être relaché de son intelligence fondée. Toute exactitude journalistique se légitime par la moralité. La philosophie de la vérité et la philosophie de la liberté donnent preuve de nécessité de la théorie de la construction de la réalité journalistique. Le journalisme a besoin d'une philosophie pratique.

\section{RESUMEN}

El libro de Walter Lippmanns ("Imagenes en nuestra Cabeza") "Pictures in our Heads" ha sido en parte una explicación de la realidad de los media. „Pero de hecho, a que se parece esta realidad! - Cuán real es en especial la televisión, con su influencia sobre los valores sociales? El autor hace la comparación de consideraciones filosóficas y aquellas de la antropología social con el periodismo. De esta manera todo el área del reconocimiento permite el trabajo de comunicación en una via muy específica. La Hermenéutica y el periodismo deben encontrarse. Al mismo tiempo uno puede ver como una teoría del reconocimiento de la realidad exige una ética del periodismo. La dimensión ética se muestra tambien en la divergencia entre técnicas y vida humana. Los instrumentos técnicos del periodismo no pueden ser separados de una inteligencia razonable.

Todo periodismo sincero se legitima a través de su ética. La filosofía de la verdad y libertad apuntan hacía una necesaria realidad periodistica. El periodismo necesita una filosofía práctica. - 